\section{Feeding Mechanisms in Anuran Tadpoles}

I cAN confirm that the method of mucous cord feeding referred to by $\operatorname{Dodd}^{\mathbf{1}}$ plays a part in the natural lives of tadpoles of Rana temporaria, Bufo bufo and Bombina variegata living under natural or semi-natural conditions. It is, however, remarkable that among the many tadpoles which have been examined, definite mucous cords have been seen in only a few. The animals appear to use this method when in the presence of fine particles; but seem to have another means of dealing with the coarse material which so often forms their food. Whole Daphnia, insect larvæ or spiders are not uncommon constituents of the food, and much exceed the diameter of the cords.

A study of the pharynx of several species of Anura has shown that the velum is in two parts, ventral and dorsal, of very different structure. The ventral velum is continuous with the floor of the pharynx, and appears to vary in elevation so that the water stream passing over it is sometimes directed at the posterior wall of the pharynx (in the centre, directly at the cesophageal opening); but in other positions of the ventral velum the water strikes the roof of the pharynx just anterior to the dorsal velum. This is a loose pocket, with the opening facing forward, and can be blown into shape by a stream of water. The relative positions of the edges of the dorsal and ventral vela are often such that the water must blow the dorsal velum open. It is in the shielded space on the downstream side of the dorsal velum that the mucous cords form; but the coarse material seems to collect more ventrally, when, indeed, it is not in the œsophageal funnel itself.

Water striking the posterior wall of the pharynx, or the cesophageal opening, must turn ventrally and then forwards through almost $180^{\circ}$ before escaping through the gill filters. It is suggested that, during this sharp turn, large objects leave the stream due to centrifugel action. The efficiency of such separa. tion increases with the size of the particles, so that the difficulty in the relative size of the cords and the food does not arise.

In a microphagous species, Chaperina fusca, the ventral velum is widely interrupted in the centre, just where in Rana and Bufo it is most strongly developed opposite the cesophagus; but the dorsal velum, which in Rana and Bufo is interrupted in the centre, is continuous in $C$. fusca, with merely a diminution in size near the central line.

The gill filters do not usually have much food upon them, and may serve merely to retain food not thrown out of the stream on the first circuit. It has always been difficult to explain how the food on the filters gets to the cesophagus against the prevailing current of water. If, however, the filters take a subsidiary role, this small amount of food may be thrown back into the pharynx by a back-wash of water from the atrium, during inspiration. The alternate passage of water containing, on both sides of the filter, suspended matter which is often gelatinous would seem to be a dangerous proceeding; but the atrium contains water which has already been filtered, and will not clog the filter on the under side,

"Rosenlaui",

R. Maxwell Savage

Parkgate Crescent,

Hadley Wood,

Barnet, Herts.

Feb. 18.

${ }^{1}$ Dodd, J. M., Nature, 165, 283 (1950).

\section{Exoerythrocytic Schizogony in Bat Malaria}

Malaria parasites of the epauletted fruit bats of tropical Africa have been described by Rodhain from the Belgian Congo, by Rousselot from French West Africa, and by myself from Kenya. Dr. R. B. Heisch, of the Division of Insect-borne Diseases, Kenya, noted the presence of a similar parasite in Epomophorus wahlbergi and was kind enough to have a number of these bats collected from near Nairobi for me when I was on a visit to the East African territories.

Seventeen bats were examined, and gametocytes were found in the blood of four. Tissues from the infected specimens were prepared for sectioning; in the liver of the most heavily infected were found the exoerythrocytic schizonts of the parasite. The smallest schizont was a spherical object about $45 \mu$ in diameter with a deeply convoluted surface, and extremely dense cytoplasm studded with nuclei. A larger form measured $70 \mu \times 55 \mu$. These immature parasites lay between the parenchyma cells, and it was impossible to determine where they had originated. After a period of further growth, the substance of the parasite appeared to divide into a large number of well-defined cytomeres enclosed within a thick wavy border of unknown origin. These cytomeres were more numerous at the periphery, where presumably final division occurs. The last stage of merocyst formation was not found, though post-mature bodies were common in the shape of foci of phagocytic cells, including giant cells surrounding and invading many free merozoites. Such foci were easily visible as tiny white opaque spots on the surface of the liver.

Sections of heart and lung revealed no schizonts. Further examination of the material will be made at a later date.

Exoerythrocytic schizogony in insectivorous bat malaria was described by Mer and Goldblum ${ }^{1}$, and has a totally different character from the appearances briefly recorded here. The latter are sufficiently striking to enable me to assign this parasite of African fruit bats to the genus Hepatocystis, the main features resembling the developmental stages of H, kochi.

I have pleasure in acknowledging the many facilities for this study provided by the Division of Insectborne Diseases, Medical Research Laboratory, Kenya. P. C. C. GarnhaM

London School of Hygiene and Tropical Medicine, Gower Street, London, W.C.1. May 31.

'Mer, C. G., and Goldblum, N., Nature, 159, 444 (1947).

\section{Failure of Antibiotics in Experimental Rabies}

As chloromycetin has shown promising results in various virus infections in man, it was decided to investigate its activity against experimentally induced rabies. Two rabbits were inoculated intracerebrally with the strain of fixed virus previously described by Kirk in Nature ${ }^{\mathrm{i}}$. The rabbits received respectively 100 and $200 \mathrm{mgm}$. of chloromycetin per kilo daily, given in three equally divided doses by mouth. As controls three rabbits were used; two received 20,000 units penicillin in procaine oil per kilo daily, and the third $40 \mathrm{mgm}$. of streptomycin per kilo daily in two equally divided doses by intra-muscular 